\title{
Estudo temporal das arboviroses: Uma análise espacial
}

\author{
Temporal study of arboviroses: A space analysis \\ Estudio temporal de las arboles: Un análisis espacial
}

Flaviana Calixta de Medeiros Silva

ORCID: https://orcid.org/0000-0001-6625-0860 Universidade Federal do Rio Grande do Norte, Brasil

E-mail: flavianapatu@ @otmail.com

Héllyda de Souza Bezerra

ORCID: https://orcid.org/0000-0002-2747-4981 Universidade Federal do Rio Grande do Norte, Brasil E-mail: hellydasbezerra@ hotmail.com

Astha Oliveira Catônio de Araújo

ORCID: https://orcid.org/0000-0002-1024-3490 Universidade Federal do Rio Grande do Norte, Brasil E-mail: asthaoliveiraar@yahoo.com.br

Lúcia Emanuelle Silva de Carvalho

ORCID: https://orcid.org/0000-0001-6171-9738 Universidade Federal do Rio Grande do Norte, Brasil E-mail: luciaemanuellec@gmail.com

José Adailton da Silva

ORCID: https://orcid.org/0000-0002-6037-7649 Universidade Federal do Rio Grande do Norte, Brasil E-mail: joseadailtonmec@gmail.com

\begin{abstract}
Resumo
O objetivo do estudo é analisar espacialmente a distribuição temporal dos casos notificados das Arboviroses nos 167 municípios do Rio Grande do Norte, nos últimos 10 anos. Trata-se de um estudo quantitativo, ecológico, descritivo, analisando espacialmente as Arboviroses Dengue, Zika e Chikungunya. Utilizou-se dados secundários disponíveis no Departamento de Informática do Sistema Único de Saúde-DATASUS, e dados disponibilizados pela Secretaria de Estado de Saúde Pública do Rio Grande do Norte-SESAP. A pesquisa foi composta pelos casos notificados das Arboviroses na população do Rio Grande do Norte, dentre os anos 2007 a 2016. Os dados foram tabulados no programa Excel versão 2013, sendo migrados posteriormente para organização e análise estatística no programa SPSS versão 22. A espacialização foi realizada no programa TABWIN versão 32. A Dengue apresenta-se a Arbovirose com maior média em 2016, possuindo maior número de notificações na sétima região de saúde do Estado, e uma rápida disseminação da Zika e Chikungunya nos últimos três anos. Na análise espacial, a distribuição apresenta-se diferenciada, dependendo da taxa de prevalência da Arbovirose, sendo a Dengue e a Chikungunya destacando municípios na região do Seridó (quarta região) e a Zika no Trairi, mais precisamente na quinta região. Conclui-se que são necessárias maiores avaliações pela vigilância epidemiológica nas regiões de maiores prevalências das Arboviroses no Estado, no intuito do fortalecimento e redirecionamento das políticas de controle destas doenças e com isso, traçar melhores estratégias preventivas e educativas direcionadas a estes agravos.
\end{abstract}

Palavras-chave: Infecções por Arboviroses; Análise espacial; Vigilância epidemiológica.

\begin{abstract}
The objective of the study is to analyze spatially the temporal distribution of the reported cases of Arboviroses in the 167 municipalities of Rio Grande do Norte, in the last 10 years. This is a quantitative, ecological, descriptive, crosssectional study, analyzing spatially the Arboviroses Dengue, Zika and Chikungunya. Secondary data were available in the SUS-DATASUS Computing Program, and data provided by the Public Health Secretariat of Rio Grande do NorteSESAP. The research was composed of the reported cases of Arbovirosis in the population of Rio Grande do Norte from 2007 to 2016. The data were tabulated in the Excel version 2013 program and later migrated to organization and statistical analysis in the SPSS version 22 program. Spatialization was performed in the TABWIN version 32 program. Dengue presents the highest Arbovirose in 2016, with the highest number of notifications in the seventh state health region, and a rapid spread of Zika and Chikungunya in the last three years. In the spatial analysis, the distribution is differentiated depending on the prevalence rate of Arbovirose, with Dengue and Chikungunya highlighting municipalities in the region of Seridó (fourth region) and Zika in Trairi, more precisely in the fifth region. It is concluded that greater evaluations by epidemiological surveillance are necessary in the regions with the
\end{abstract}


highest prevalence of Arbovirosis in the State, in order to strengthen and redirect policies to control these diseases and with that, to draw up better preventive and educational strategies directed to these diseases.

Keywords: Arbovirus infections; Spatial analysis; Epidemiological surveillance.

\begin{abstract}
Resumen
El objetivo del estudio es analizar espacialmente la distribución temporal de los casos notificados de las Arbovirosis en los 167 municipios de Rio Grande do Norte, en los últimos 10 años. Se trata de un estudio cuantitativo, ecológico, descriptivo, transversal, analizando espacialmente las Arbovirosis Dengue, Zika y Chikungunya. Se utilizaron datos secundarios disponibles en el Programa de Informática del SUS-DATASUS, y datos disponibilizados por la secretaría de salud Pública de Rio Grande do Norte-SESAP. La encuesta fue compuesta por los casos notificados de las Arbovirosis en la población de Rio Grande do Norte, entre los años 2007 a 2016. Los datos fueron tabulados en el programa Excel versión 2013, siendo migrados posteriormente para organización y análisis estadístico en el programa SPSS versión 22. La espacialización se realizó en el programa TABWIN versión 32. El Dengue se presenta a Arbovirosa con mayor promedio en 2016, con mayor número de notificaciones en la séptima región de salud del Estado, y una rápida diseminación de Zika y Chikungunya en los últimos tres años. En el análisis espacial, la distribución se presenta diferenciada, dependiendo de la tasa de prevalencia de la Arbovirosa, siendo la Dengue y la Chikungunya destacando municipios en la región del Seridó (cuarta región) y la Zika en el Trairi, más precisamente en la quinta región. Se concluye que son necesarias mayores evaluaciones por la vigilancia epidemiológica en las regiones de mayores prevalencias de las Arbovirosis en el Estado, con el fin del fortalecimiento y redireccionamiento de las políticas de control de estas enfermedades y con ello, trazar mejores estrategias preventivas y educativas dirigidas a estos agravios.
\end{abstract}

Palabras clave: Infecciones por Arbovirosis; Análisis espacial; Vigilancia epidemiológica.

\title{
1. Introdução
}

A grande maioria dos patógenos responsáveis pelas doenças infecciosas que afetam os seres humanos tem origem zoonótica, e com a modificação do ambiente causada por ações antrópicas, tem a sua transmissão ao homem favorecida. Nos últimos 10 anos, observa-se a emergência de algumas doenças transmitidas por mosquito vetores, em especial as Arboviroses (Lima-Camara, 2016; Lopes, Nozawa \& Linhares, 2014).

As Arboviroses manifestam-se clinicamente nos seres humanos como doença febril indiferenciada, moderada ou grave, erupções cutâneas, artralgia, a síndrome neurológica e a síndrome hemorrágica. A artralgia manifesta-se como exantema ou rash maculopapular, poliartrite e poliartralgia; a síndrome hemorrágica é evidenciada por petéquias, hemorragia e choque combinado com uma redução intensa de plaquetas; as síndromes neurológicas podem manifestar-se como mielite, meningite e/ou encefalite, com mudanças de comportamento, paralisia, paresia, convulsões e problemas de coordenação; e a doença febril apresenta-se com sintomas de gripe (febre, cefaleia, dor retro-orbital e mialgia) (Lopes et al., 2014).

Transmitidos por mosquitos vetores amplamente distribuídos ao longo do território brasileiro (Aedes aegypti e Aedes albopictus) e associados ao homem, a população encontra-se expostas à infecção pelos Arbovírus causadores da Dengue (DENV), Chikungunya (CHIKV) e Zika (ZIKV). No Brasil, essas espécies foram introduzidas em diferentes momentos: Ae. aegypti no período da colonização e Ae. albopictus no final do século XX (Júnior, Serpa, Barbosa, Pereira, Holcamn, Voltolini \& Marques, 2019). Há evidências que sugerem que a dispersão e/ou manutenções dessas doenças está estreitamente relacionadas à precariedade dos serviços de saneamento básico e abastecimento de água (Cavalcanti \& Timerman, 2016).

Desse modo, as Arboviroses têm se mostrado um desafio à saúde pública, significando uma potencial ameaça que tem emergido e/ou reemergido nas últimas décadas à saúde humana. No Brasil, o controle dessas doenças está baseado em um conjunto de ações ligadas à vigilância em saúde, buscando estratégias que tem como objetivos a análise permanente da situação de saúde das populações e o desenvolvimento adequando de práticas que permitam o enfrentamento dos problemas existentes (Lima-Camara, 2016; Cavalcanti \& Timerman, 2016). A prevenção e o controle da dengue tornaram-se tarefas difíceis, devido à força de morbidade do agente infeccioso, à alta competência vetorial do Aedes aegypti e ao elevado custo de execução das ações de controle vetorial, além das implicações desfavoráveis associadas ao uso de inseticidas no meio ambiente 
(Barbosa, Tavares, Torres, Nascimento, Moura, Vieira, Araújo, \& Gama, 2017).

Dentre as doenças transmitidas por mosquito vetores destacam-se atualmente em especial Arboviroses como Chikungunya, Zika e Dengue em diferentes países da América que, em geral, circulam entre os animais silvestres, com alguma especificidade por hospedeiros e mantendo-se em ciclos enzoóticos em poucas espécies de vertebrados e invertebrados. O homem ou animais domésticos geralmente são hospedeiros acidentais, ocasionados (Donalisio, Freitas, Zuben, 2017) por modificações dos ecossistemas pelo homem, crescimento populacional urbano desordenado e ampliação do intercâmbio internacional. Ressalta-se que em países já endêmicos para a Dengue, como o Brasil, pode ter como consequência o colapso dos serviços de saúde em caso de epidemias simultâneas (Lima-Camara, 2016; Lopes et al., 2014). Visto que, o custo associado ao manejo da dengue no Brasil é considerado o maior das Américas, correspondendo a $42 \%$ dos gastos totais relacionados à doença no continente (Teich, Arinelli, Fahham, 2017).

Estabelecer essa relação, entre ambiente e saúde, é importante para que haja maior prevenção desses agravos à saúde, entendendo que para o controle de vetores de doenças em áreas que apresentaram rápida urbanização são necessárias não somente ações de saúde, mas que hajam políticas que integrem a mobilização da sociedade, saúde, educação ambiental, melhorias de habitação, saneamento e ações para evitar mais desmatamento (Almeida, Cota, Rodrigues, 2020).

Os Arbovírus são causadores de doenças em humanos e em outros animais de sangue quente e se reproduzem nos tecidos de insetos hematófagos. Ocorrem em áreas tropicais e subtropicais de todos os continentes, tendo por hospedeiros vertebrados susceptíveis, nos quais provocam viremias capazes de infectar um novo hospedeiro invertebrado (Lima-Camara, 2016; Lopes et al., 2014).

Os impactos econômicos dessas novas Arboviroses são preocupantes, pois apesar de a maioria dos pacientes infectados apresentarem recuperação completa após a fase aguda da doença, alguns sintomas como a forte artralgia do CHIKV podem durar semanas ou meses. Por outro lado, a infecção por ZIKV pode levar o paciente a desenvolver a síndrome de Guillain-Barré, uma síndrome de origem autoimune e de ordem neurológica que causa fraqueza muscular generalizada e paralisia. Além disso, a Zica está associada ao recente surto de microcefalia em bebês recém-nascidos no Brasil, o que aumenta a urgente necessidade de implementar a vigilância em saúde relacionada a essa infecção (Lima-Camara, 2016).

O controle e a prevenção de Arboviroses no Brasil têm se mostrado ineficazes no combate a estas enfermidades, em parte, decorrente do uso indiscriminado de medicamentos e inseticidas que proporcionam o desenvolvimento de resistência por parte dos agentes etiológicos e insetos vetores. Observa-se a necessidade da busca por novas estratégias como alternativas à prevenção e controle da disseminação dessas doenças (Maniero et al, 2016).

Tendo em vista o atual contexto das Arboviroses no Brasil, o presente estudo mostra-se relevante na medida em que pretende realizar uma análise espacial dos casos de Dengue, Chikungunya e Zika notificados no Estado do Rio Grande do Norte nos últimos 10 anos. A análise do estudo contribuirá com o conhecimento epidemiológico necessário para o fortalecimento e redirecionamento das políticas de controle das Arboviroses e com isso, traçar melhores estratégias preventivas e educativas direcionadas a esses agravos.

O presente estudo busca analisar espacialmente a distribuição temporal dos casos notificados de Arboviroses nos 167 municípios do Rio Grande do Norte, nos últimos 10 anos.

\section{Metodologia}

Trata-se de um estudo quantitativo, ecológico, exploratório e descritivo, no qual analisa espacialmente as Arboviroses Dengue, Zika e Chikungunya, utilizando-se dados secundários. Nesta abordagem, o interesse é no coletivo, naquilo que pode ser predominante como característica do grupo. Essa dinâmica promove em seu interior o fortalecimento da estatística, diante 
da sua potencialidade na análise de dados e possibilidade de inferência (Mussi, Mussi, Assunção \& Nunes, 2019). A pesquisa foi realizada a partir de dados extraídos do Departamento de Informática do Sistema Único de Saúde (DATASUS) para os anos de 2007 a 2012, e pela Secretaria de Estado da Saúde Pública do Rio Grande do Norte (SESAP-RN) para os anos de 2013 a 2016, período não disponibilizado pelo DATASUS. No entanto, para as Arboviroses Zika e Chikungunya, as notificações só constam a partir de 2014 .

O universo da pesquisa foi composto pelos 167 municípios do Estado do Rio Grande do Norte que tiveram seus dados associados a notificações de casos de Dengue, Zika e Chikungunya nos últimos 10 anos. Para melhor análise, também se levou em consideração as oito regiões de saúde do Estado do Rio Grande do Norte, respectivamente: Litoral Sul e Agreste, Oeste, Mato Grande e Salineira, Seridó, Trairi e Potengi, Alto Oeste, Metropolitana e Vale do Açu. As variáveis utilizadas na pesquisa foram o número de casos notificados, município de notificação e o sexo dos indivíduos.

Optou-se pela realização do estudo no Rio Grande do Norte, devido seus indicadores demonstrarem considerável número de casos notificados de Arboviroses, logo necessita de melhores estratégias preventivas e seguimento clínico para redução da morbidade e mortalidade no estado. Segundo dados do Instituto Brasileiro de Geografia e Estatística [IBGE] (2016), estima-se que a população do Rio Grande do Norte totalize 3.474 .998 no ano de 2016, distribuída em 167 municípios em uma área de 52.811,110 Km2. O estado é o $16^{\circ}$ colocado na lista dos estados com maior Indice de Desenvolvimento Humano Municipal (IDHM) do Brasil, com o índice de 0,684, enquanto que a média do Brasil é de 0,727.

Os dados foram inicialmente alocados no programa Microsoft Excel versão 2013, posteriormente foram processados em banco de dados do programa SPSS (Statistical Package for the Social Sciences), versão 22.0 com número de série 10101141047. Para análise dos dados foi utilizada a estatística descritiva por meio de medidas de tendência central (médias) e frequências absolutas das oito regiões de saúde. O mapeamento dos resultados foi feito através do programa de código aberto TAB para Windows - TabWin 32, criado pelo DATASUS, no qual foi calculada a taxa de prevalência, e construído um mapa para cada Arbovirose. O Tabwin é um tabulador desenvolvido pelo DATASUS para ser utilizado nas bases de dados (SUS).

\section{Resultados}

A amostra do presente estudo foi composta pelos 167 (100\%) municípios do Rio Grande do Norte (RN), divididos em oito regiões de saúde, sendo a sexta região (Alto Oeste) a maior composta dor 36 municípios, correspondendo a $21 \%$ do Estado.

Na tabela 01, no que diz respeito a média dos casos de Dengue. Esta teve uma variação considerável entre as regiões de saúde do Estado, merecendo destaque o ano de 2009 que apresentou a menor média de notificações dos últimos 10 anos, estando todas abaixo de 13, excetuando-se a sétima região com 387,0 que apesar de apresentar apenas 5 municípios em sua composição, apresenta o maior contingente populacional em relação as demais.

Ainda em relação a tabela 01, observa-se que a partir de 2011 há um aumento substancial das médias, mostrando um aumento dos números de casos em praticamente todas as regiões de saúde, com algumas quedas e picos ao logo dos anos, culminando com o ano de 2016, que apresenta as maiores médias dos últimos 10 anos para a ocorrência de casos de Dengue notificados. 
Tabela 1. Série temporal da notificação de Dengue no Estado do Rio Grande do Norte, de 2007 a 2016.

\begin{tabular}{l|c|c|c|c|c|c|c|c|c|c|c} 
& $\mathbf{N}$ & $\begin{array}{c}\text { Media } \\
\mathbf{2 0 0 7}\end{array}$ & $\begin{array}{c}\text { Média } \\
\mathbf{2 0 0 8}\end{array}$ & $\begin{array}{c}\text { Média } \\
\mathbf{2 0 0 9}\end{array}$ & $\begin{array}{c}\text { Média } \\
\mathbf{2 0 1 0}\end{array}$ & $\begin{array}{c}\text { Média } \\
\mathbf{2 0 1 1}\end{array}$ & $\begin{array}{c}\text { Média } \\
\mathbf{2 0 1 2}\end{array}$ & $\begin{array}{c}\text { Média } \\
\mathbf{2 0 1 3}\end{array}$ & $\begin{array}{c}\text { Média } \\
\mathbf{2 0 1 4}\end{array}$ & $\begin{array}{c}\text { Média } \\
\mathbf{2 0 1 5}\end{array}$ & $\begin{array}{c}\text { Média } \\
\mathbf{2 0 1 6}\end{array}$ \\
\hline $\begin{array}{l}\text { Região } \\
\mathbf{1}\end{array}$ & 27 & 37,96 & 29,85 & 2,03 & 6,18 & 57,77 & 50,70 & 72,85 & 72,00 & 70,70 & 79,63 \\
\hline $\begin{array}{l}\text { Região } \\
\mathbf{2}\end{array}$ & 16 & 18,88 & 120,81 & 12,13 & 18,19 & 126,19 & 119,69 & 55,88 & 50,00 & 176,12 & 333,25 \\
\hline $\begin{array}{l}\text { Região } \\
\mathbf{3}\end{array}$ & 25 & 27,20 & 90,56 & 3,08 & 5,76 & 73,64 & 8,96 & 40,72 & 27,08 & 85,92 & 444 \\
\hline $\begin{array}{l}\text { Região } \\
\mathbf{4}\end{array}$ & 25 & 23,24 & 66,32 & 4,68 & 12,28 & 33,22 & 85,20 & 243,56 & 147,12 & 183,52 & 488,28 \\
\hline $\begin{array}{l}\text { Região } \\
\mathbf{5}\end{array}$ & 21 & 52,33 & 103,90 & 9,62 & 13,67 & 75,15 & 40,67 & 160,10 & 56,33 & 122,71 & 182,09 \\
\hline $\begin{array}{l}\text { Região } \\
\mathbf{6}\end{array}$ & 36 & 13,97 & 81,53 & 1,67 & 24,97 & 32,92 & 57,28 & 111,89 & 52,97 & 30,41 & 211,30 \\
\hline $\begin{array}{l}\text { Região } \\
\mathbf{7}\end{array}$ & 5 & 1740,40 & 3264,00 & 387,0 & 1382,0 & 2292,6 & 2981,4 & 1491,8 & 1073,6 & 2243,0 & 3537,8 \\
\hline $\begin{array}{l}\text { Região } \\
\mathbf{8}\end{array}$ & 12 & 6,83 & 51,83 & 5,25 & 2,66 & 25,50 & 60,75 & 19,00 & 15,50 & 44,91 & 133,66 \\
\end{tabular}

Fonte: DATASUS/2017

No tocante a tabela 02, esta diz respeito a média dos casos de Zika e Chikungunya. Pode-se observar que no território do Rio Grande do Norte apenas a partir de 2014 é que ocorreram casos notificados dessas Arboviroses, destacando as regiões de saúde 4 e 7, no qual foram as únicas com notificações, com médias respectivas de 0,2 e 3,6.

Ainda na tabela 02 , no ano 2015 pode-se observar a presença de casos notificados em todas as regiões de saúde do Estado, encontrando-se a menor média na Região 6 (Alto Oeste), apresentando valor de 1,1 e a maior média na Região 7 (Região Metropolitana) com valor de 254,2. Observa-se ainda a tendência crescente para o ano de 2016, em que todas as regiões de saúde apresentam aumento nas médias dos casos notificados de Zika e Chikungunya desde a entrada das doenças em território nacional no ano de 2014.

Tabela 2. Série temporal das notificações Zika e Chikungunya no Estado do Rio Grande do Norte, de 2014 a 2016.

\begin{tabular}{lllll}
\hline & $\mathrm{N}$ & \multicolumn{3}{c}{ Média } \\
\cline { 3 - 5 } & & 2014 & 2015 & 2016 \\
\hline Região 1 & 27 & 0,0 & 2,62 & 12,81 \\
Região 2 & 16 & 0,0 & 8,68 & 130,06 \\
Região 3 & 25 & 0,0 & 16,76 & 31,08 \\
Região 4 & 25 & 0,2 & 7,36 & 43,4 \\
Região 5 & 21 & 0,0 & 7,38 & 45,42 \\
Região 6 & 36 & 0,0 & 1,1 & 27,63 \\
Região 7 & 5 & 3,6 & 254,2 & 829,8 \\
Região 8 & 12 & 0,0 & 6,5 & 19,5 \\
\hline
\end{tabular}

Fonte: SESAP-RN/2017. 
A Tabela 3 diz respeito ao número absoluto de notificações de Arboviroses relacionado ao sexo no Estado do Rio Grande do Norte de 2014 a 2016. O que observamos é que nas três Arboviroses, mais da metade dos indivíduos que notificaram a infecção são do sexo feminino.

Tabela 3. Número absoluto e frequência de notificações das Arboviroses relacionado ao sexo no Estado do Rio Grande do Norte, de 20014 a 2016.

\begin{tabular}{|c|c|c|c|}
\hline \multirow[t]{3}{*}{ Arboviroses } & \multicolumn{2}{|c|}{ Sexo } & \multirow{3}{*}{$\begin{array}{c}\text { Total de casos } \\
\text { notificados }\end{array}$} \\
\hline & Feminino & Masculino & \\
\hline & $\mathrm{N}(\%)$ & $\mathrm{N}(\%)$ & \\
\hline Dengue & $61900(58,6 \%)$ & $43726(41,4 \%)$ & 105626 \\
\hline Zika & $3339(69,6 \%)$ & $1463(30,4 \%)$ & 4802 \\
\hline Chikungunya & $5260(64,9 \%)$ & $2834(35,1 \%)$ & 8094 \\
\hline
\end{tabular}

Fonte: DATASUS/ SESAP - RN (2017).

A Figura 1 apresenta a distribuição espacial da taxa de prevalência da Dengue por 10.000 pessoas de 2007 a 2016. Pode-se observar que todos os municípios do Estado do Rio Grande do Norte apresentam notificações de casos de Dengue, sendo a maioria dos municípios com notificações que variam de 100 a 1659 casos.

Destaca-se a $4^{\text {a }}$ Região de Saúde (Seridó) por apresentar o maior número de municípios com notificações que variam de 1659 a 2477 casos por 10.000 pessoas, sendo eles Timbaúba dos Batistas com 2.126 casos, São José do Seridó com 1670 casos, Jardim do Seridó com 2.075 casos, Santana do Seridó com 1.754 casos, Carnaúba dos Dantas com 1786 casos e Parelhas com 2.116 casos notificados, destacando-se o município de Cruzeta que apresentou 2.856 notificações.

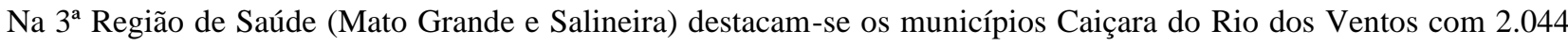
casos e Galinhos com taxa de prevalência de 1659 a 2477 casos por 10.000 pessoas, e Guamaré com 3.128 casos. Na $5^{\text {a }}$ Região de Saúde (Trairi) destaca-se Santa Cruz com 1.745 casos notificados. Na 6 ${ }^{\text {a }}$ Região de Saúde (Alto Oeste) destaca-se Pau dos Ferros com 4.114 casos. 
Research, Society and Development, v. 10, n. 7, e10910716220, 2021

(CC BY 4.0) | ISSN 2525-3409 | DOI: http://dx.doi.org/10.33448/rsd-v10i7.16220

Figura 1 - Taxa de prevalência da Dengue por 10.000 pessoas no Estado do Rio Grande do Norte, 2007 a 2016.

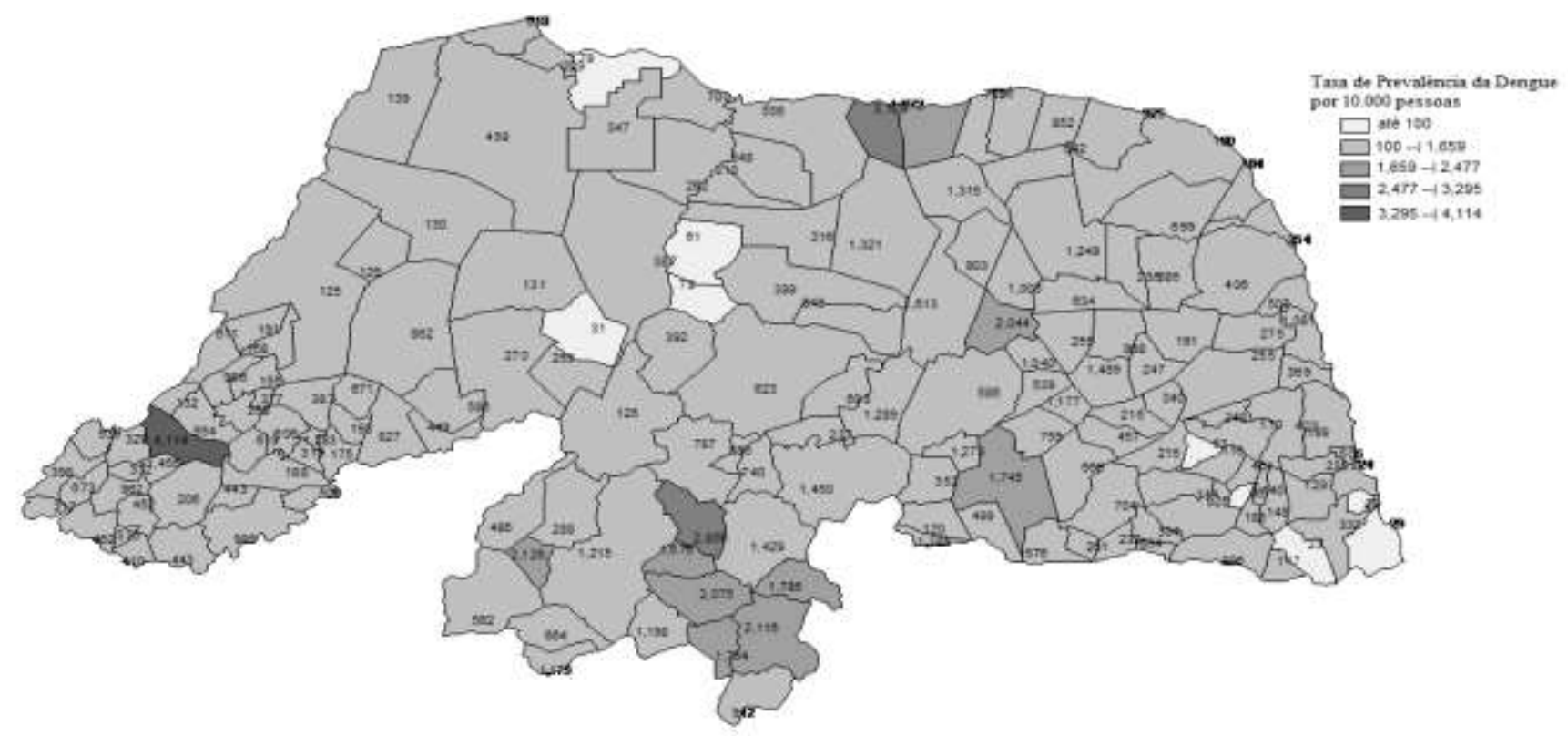

Fonte: DATASUS/SESAP-RN (2017).

A Figura 2 trata da taxa de prevalência de Chikungunya por 10.000 pessoas, tendo por base os casos notificados no Rio Grande do Norte de 2014 a 2016. Observa-se no mapa que até 2016 ainda existiam 51 municípios no Estado que não haviam apresentado notificações de casos de Febre Chikungunya. Os demais municípios apresentavam-se dentro da faixa de prevalência de 0 a 171 casos. Pode-se destacar ainda o município de Rafael Godeiro e Patú da 6 Região de Saúde (Alto Oeste) com 428 e 257 casos respectivamente, o município de São José do Seridó da $4^{\text {a }}$ Região de Saúde (Seridó) com 206 casos, e o município de Taipú na $3^{\mathrm{a}}$ Região de Saúde (Mato Grande e Salineira) com 288 casos de Chikungunya. 
Research, Society and Development, v. 10, n. 7, e10910716220, 2021

(CC BY 4.0) | ISSN 2525-3409 | DOI: http://dx.doi.org/10.33448/rsd-v10i7.16220

Figura 2 - Taxa de Prevalência da Chikungunya por 10.000 pessoas no Estado do Rio Grande do Norte, 2014 a 2016.

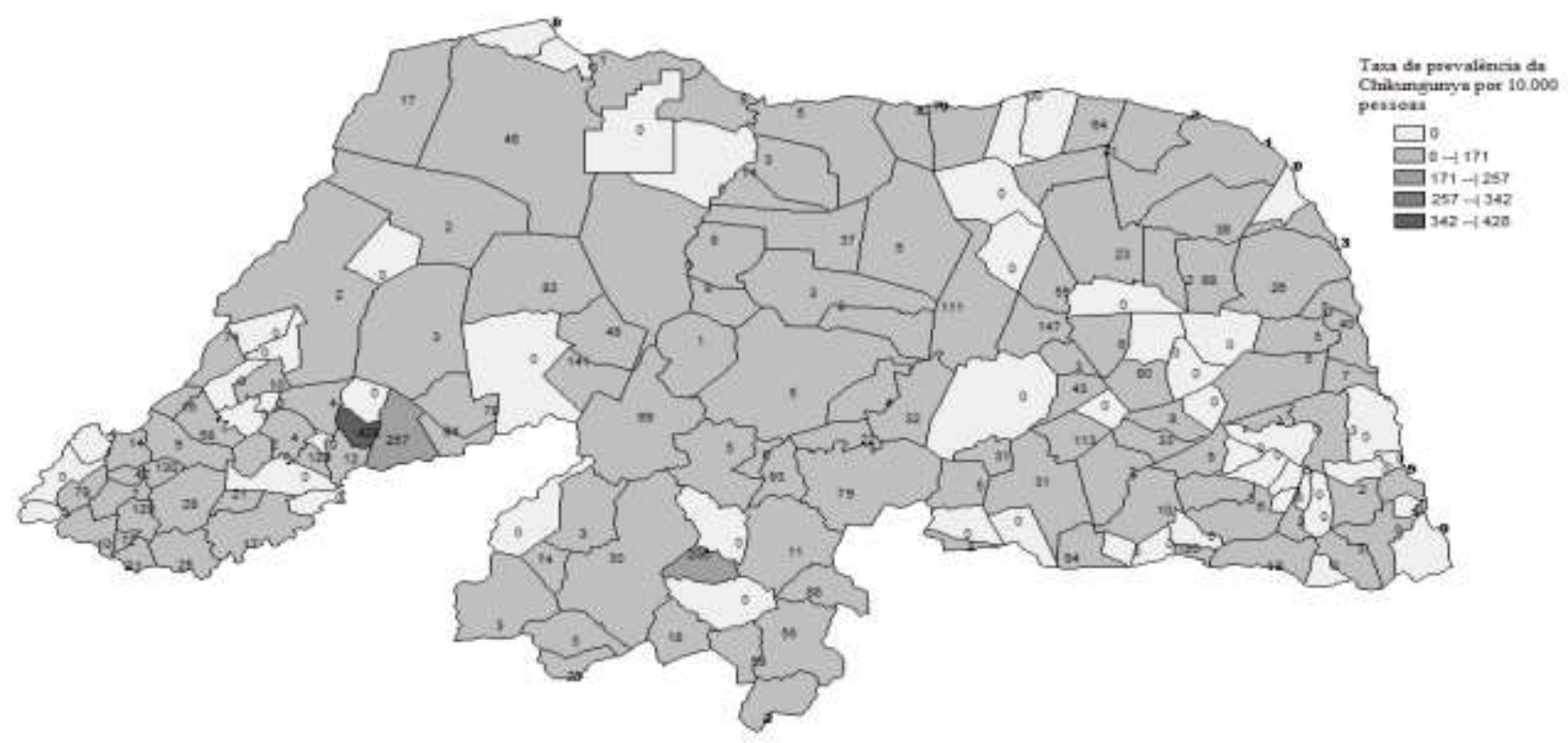

Fonte: DATASUS/SESAP-RN (2017).

A Figura 3 retrata a taxa de prevalência da Zika por 10.000 pessoas do ano de 2014 ao ano de 2016, que são os anos em que se tem registro da ocorrência de casos da doença no território brasileiro. Observa-se que dos 167 municípios do Estado do Rio Grande do Norte, 90 ainda não apresentavam notificações da doença até 2016. Os outros 74 municípios apresentavam taxa de prevalência abaixo dos 81 casos da doença, com destaque para Extremoz com 90 casos notificados na $7^{\text {a }}$ Região de Saúde (Metropolitana), e Elói de Souza e Santa Cruz da 5a Região de Saúde (Trairi) com 112 e 135 casos respectivamente. 
Figura 3 - Taxa de prevalência da Zika por 10.000 pessoas no Estado do Rio Grande do Norte, 2014 a 2016.

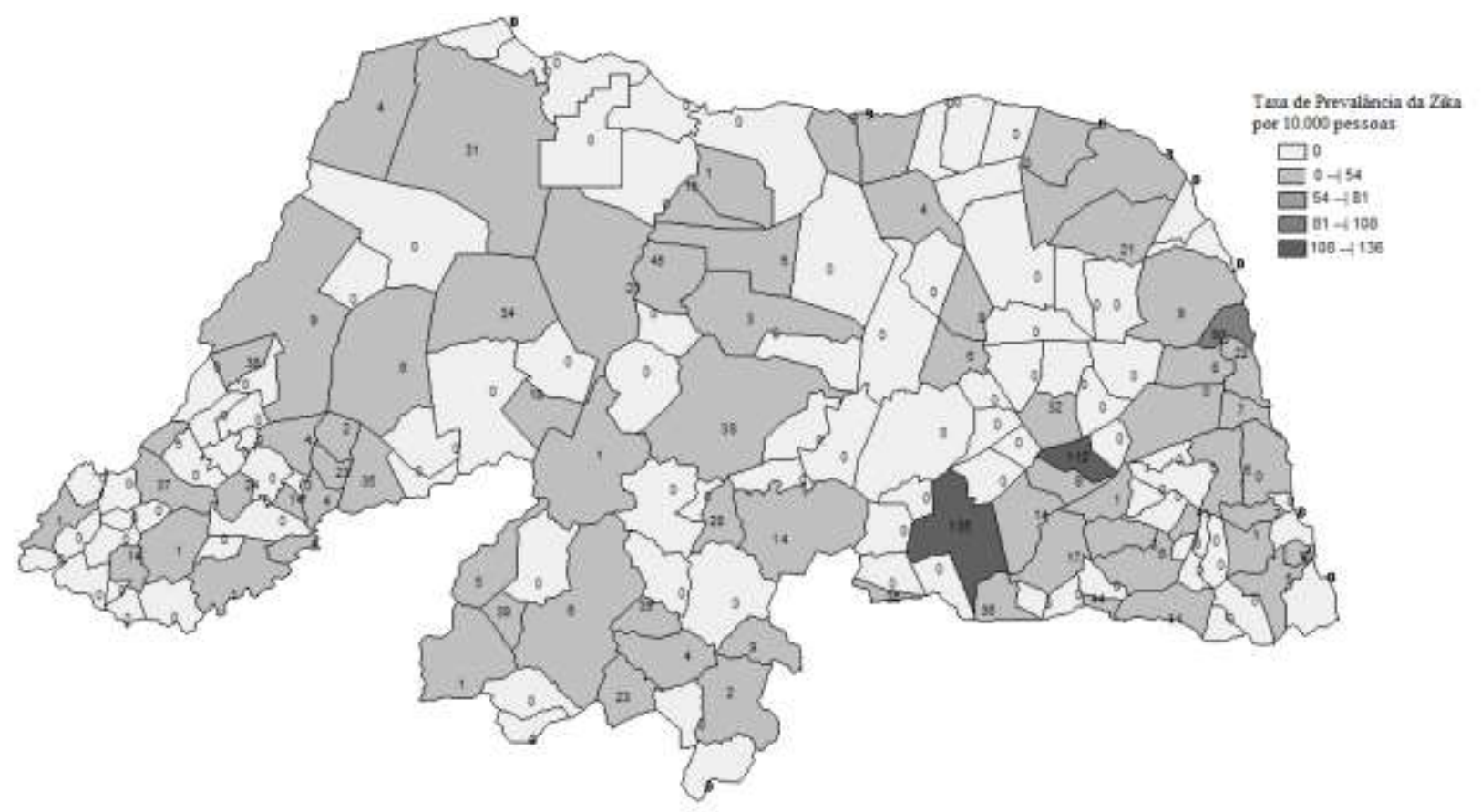

Fonte: DATASUS/SESAP-RN (2017).

\section{Discussão}

A Secretaria de Saúde Pública do Estado do Rio Grande do Norte (2016) publicou no final de 2016 um boletim epidemiológico a respeito das Arboviroses no Estado, referente às informações atualizadas até a $52^{\mathrm{a}}$ semana epidemiológica de 2016, sobre o monitoramento dos casos de Dengue, Chikungunya e Zika. O levantamento do índice de infestação pelo mosquito Aedes aegypti aponta que 73 municípios encontravam-se em situação de alerta e 62 municípios em risco, representando 80,84\% dos municípios do Estado.

Ao longo das últimas décadas, estudos demonstram que o processo de domiciliação de insetos está correlacionado à urbanização acelerada das cidades, possibilitando a dispersão de pragas e transmissores de doenças, causando transtornos socioeconômicos à sociedade, em consequência dos problemas de saúde (Alencar et al., 2008).

A Dengue é a Arbovirose mais importante do mundo e cerca de 2,5 bilhões de pessoas encontravam-se em condições susceptíveis à infecção até o ano de 2009. Esse risco se torna maior em países que apresentam clima tropical, como o Brasil, onde a temperatura e as distribuições das chuvas favorecem a proliferação do mosquito vetor. É uma doença infecciosa febril aguda causada pelo vírus DENV da família Flaviviridae e do gênero Flavivirus, podendo manifestar-se como uma síndrome viral benigna ou um quadro grave de uma doença hemorrágica (Almeida, Medronho, \& Valencia, 2009; Nakagawa, 2013).

No Brasil, já havia relatos da Dengue desde o século XIX e desde 1976 o mosquito Aedes aegypti foi reintroduzido no país e estava presente em diversos países da América Látina, no entanto casos da doença só foram confirmados em 1982 quando foram identificados dois tipos de vírus (DENV-1 e DENV-4). Existem hoje quatro tipos conhecidos de vírus causador da doença, que variam conforme os sorotipos: DEN-1, DEN-2, DEN-3 e DEN-4, não havendo a imunização cruzada (Nakagawa, 2013). 
Em relação a tabela 01, foi visto que de 2007 a 2016 a média dos casos de Dengue em cada uma das 8 regiões de saúde se apresentou de forma flutuante, onde pode-se observar anos com diminuição dos casos seguidos por anos onde houve o aumento, culminando com 2016, que foi o ano em que se observou a maior média de casos da Arbovirose da década analisada. Esses picos e baixas observados no Rio Grande do Norte quanto ao comportamento dos casos de Dengue também ocorreu em outros Estados e regiões do país11.

A deficiente distribuição dos serviços de saúde e o insuficiente investimento na prevenção e no tratamento adequados e de forma descontinua, ocasionam a seguinte situação que pode explicar a flutuação das taxas de casos: quando um ano tem redução dos índices de casos, diminui-se a atenção e investimentos, resultando no aumento dos casos no ano seguinte. Além disso a deficiência nos sistemas de abastecimento, distribuições irregulares das chuvas, assim como crises hídricas, como a ocorrida em 2016, obrigam a população a manter em domicílio reservatórios de água, muitas vezes inadequados, o que facilita a deposição de ovos e o desenvolvimento de mais mosquitos vetores11,12. Para Fuck (2010), o controle da dengue obteria maior sucesso com a implementação de ações educativas, sendo essas de extrema importância como formas de fortalecer as ações já desenvolvidas e criar meios alternativos de controle.

A mesma situação relativa a proliferação dos casos de Dengue colabora para a disseminação da febre Chikungunya e da Zika, dado o fato de que o Aedes aegypti também se constituir vetor para a transmissão das três Arboviroses em território nacional.

A febre do chikungunya é uma Arbovirose causada pelo vírus Chikungunya (CHIKV), da família Togaviridae e do gênero Alphavirus. O primeiro registro de transmissão autóctone no Brasil ocorreu em setembro de 2014, na cidade de Oiapoque (Amapá), e atualmente este Estado, Bahia e Pernambuco são os que mais notificam casos no país. Ao longo de 2014 foram confirmados 2.772 casos de CHIKV e em 2015, até a $12^{\mathrm{a}}$ semana epidemiológica foram confirmados 1.513 casos autóctones (Lima-Camara, 2016)13.

O Zika vírus, também pertencente à família Flaviviridae e ao gênero Flavivirus, foi isolado primeiramente em macacos rhesus em 1947, na Floresta Zika na Uganda. Uma epidemia de Zika atingiu a ilha Yap, Micronésia, no Oceano Pacífico, enquanto que a Polinésia Francesa, também na Oceania, registrou uma grande epidemia da doença em outubro de 2013, assim confirmando a circulação e a transmissão viral fora dos continentes africano e asiático1 (Lima-Camara, 2016).

De acordo com a Comissão de Epidemiologia da ABRASCO14, ao final de 2014, foi identificado um surto de uma doença exantemática febril de etiologia desconhecida no Rio Grande do Norte. Em maio de 2015, o vírus Zika que até então não havia circulado na América Continental, foi confirmado por testes de RT-PCR em 16 de 46 amostras da Bahia e Rio grande do Norte. Em um curto espaço de tempo novos casos foram confirmados em diferentes Estados brasileiros e outros países da América Látina, Caribe, além de EUA e Europa.

A tabela 02 reflete a introdução dos casos de Zika e Chikungunya em 2014 e sua rápida disseminação nos dois anos seguintes nas regiões de saúde do Rio Grande do Norte. O que pode-se destacar a partir da tabela é a grande variação das médias de casos nas oito regiões de saúde, partindo-se de uma média, calculada com base nos dados da tabela, de 0,475 casos em 2014 para 142,46 casos em 2016, ou seja, um aumento no número de casos que gira em torno de 29991,58\%, sendo um aumento bastante alarmante.

O fator ambiental desempenha importante influência na população de mosquitos, que geralmente habitam em ambientes peridomiciliares. Isso explica o fato das maiores médias de casos das três arboviroses (Dengue, Zika e Chikungunya) se darem na $7^{\mathrm{a}}$ Região de Saúde (Metropolitana), que apesar de possuir apenas 5 municípios, detém grandes aglomerados populacionais e clima tropical propício, que favorecem uma maior presença do vetor, e consequentemente maior quantidade de casos 11,12 . 
Já a $1^{\text {a }}$ Região de Saúde composta por 27 municípios, apesar da proximidade em relação a $7^{\mathrm{a}}$ Região e clima semelhante, devido possuir uma densidade populacional menor segundo o IBGE (2016)5, apresenta as menores médias de casos de Zika e Chikungunya, até o ano de 2016.

A tabela 03 traz o número de casos de Dengue, Zika e Chikungunya e o sexo dos indivíduos acometidos. Observa-se que as Arboviroses acometem pessoas de ambos os sexos, contudo o estudo mostra maior incidência entre as mulheres do que entre os homens. A hipótese mais provável para essa ocorrência é o fato de que habitualmente, as mulheres permanecem mais tempo no intra e peridomicilio, especialmente no período diurno quando a transmissão ocorre com maior frequência, além de terem os casos mais notificados por procurarem os serviços de saúde com mais frequência11.

Do número absoluto dos casos das três Arboviroses em questão, aproximadamente 59,48\% foram do sexo feminino. De acordo com esses dados, observa-se a necessidade de se desenvolver ações de educação em saúde com este público, especialmente na atenção primária, no intuito de disseminar medidas de cunho preventivo para esta parcela da população, que é mais atingida.

As Figuras de 1 a 3 que trazem as taxas de prevalência da Dengue, Chikungunya e Zika por 10.000 pessoas, respectivamente, mostram que todas as cidades do Rio Grande do Norte apresentaram notificações de casos de Dengue nos últimos 10 anos, enquanto que de 2014 a 2016, 46,11\% dos municípios tiveram casos notificados de Zika e 69,46\% de Chikungunya. Esses dados apontam para o fato de quase $50 \%$ dos municípios do Estado apresentarem a co-circulação das três Arboviroses em seu território, o que deve ser motivo de alerta para a possibilidade da co-infecção destas doenças.

Através da Figura 1, que traz a distribuição espacial da taxa de prevalência da Dengue por 10.000 pessoas no Rio Grande do Norte entre os anos de 2007 e 2016, podemos observar que a $4^{\text {a }}$ Região (Seridó) apresenta o maior número de municípios com a taxa de prevalência maior que 1659 casos.

Podemos tentar atribuir esse fato principalmente as condições climáticas da região que favorecem longos períodos de seca durante o ano e chuvas irregulares, o que força a população a realizar o armazenamento de água potável em recipientes. O armazenamento muitas vezes incorreto favorece a disseminação dos criadouros do mosquito Aedes Aegypit e consequentemente uma maior propagação dos casos de Dengue11.

Na Figura 2 observamos a distribuição espacial da taxa de prevalência da Chicungunya por 10.000 pessoas no Estado entre os anos de 2014 e 2016. Analisa-se que em apenas 3 anos a Arbovirose alcançou casos em 116 municípios do Estado, estando presente em todas as regiões de saúde. Isso mostra uma rápida disseminação da doença, o que merece uma atenção especial dado o fato de que a Febre Chikungunya. Esta também destaca espacialmente regiões climáticas secas, como a $4^{\mathrm{a}}$ e $6^{\mathrm{a}}$ região de saúde, favorecendo mais uma vez, o acúmulo de água. Das 3 arboviroses abordadas no estudo, a Chikungunya é a que mais deixa sequelas nos indivíduos acometidos, principalmente na parcela mais idosa da população. Significa dizer que a doença continua impactando na saúde da população e nas demandas dos serviços de saúde, mesmo após a cura da virose12.

A Figura 3 traz a taxa de prevalência da Zika por 10000 pessoas no Rio Grande do Norte também entre os anos de 2014 e 2016. O que observamos é que os 4802 casos notificados da arbovirose (observados na tabela 3) se distribuem em 77 municípios do Estado, com maior prevalência na $7^{\text {a }}$ região de saúde. Destaca-se que de acordo com a Comissão de Epidemiologia da ABRASCO14, a confirmação de casos de ZIKA no Brasil, especificamente na Bahia e no Rio Grande do Norte, só se deu em maio de 2015, temos então que a disseminação dos casos da Arbovirose em 46,11 \% dos municípios do Estado se deu em pouco mais de 1 ano e meio. Além disso, esta Arbovirose por estar presente de forma espacial mais presente na região Metropolitana de saúde, na qual possui um grande aglomerado populacional, consegue se disseminar mais rapidamente. 
Em um estudo recente sobre o Zika Vírus no Brasil15, traz que Natal, que se encontra na sétima região de saúde do Rio Grande do Norte, foi uma das primeiras cidades em 2014 a ser encontrada a doença na região do Nordeste, assim como João Pessoa no Estado da Paraíba, confirmando dados presentes nesta pesquisa.

Deve-se ter em mente que apesar da Zika apresentar sintomatologia mais branda que as outras Arboviroses abordadas, a interação entre estas, poderia teoricamente resultar em viremias mais intensas ou outras alterações imunológicas capazes de se constituir em gatilhos para o desenvolvimento de doenças autoimunes, como por exemplo a Síndrome de Guillain-Barré. Destaca-se também que, embora a microcefalia esteja vinculada a muitas exposições ambientais e genéticas, entre outras, a infecção durante a gravidez pelo vírus da Zika tem acompanhado o aumento de casos de microcefalia e outras malformações neurológicas desde 2015 no Brasil14.

Pensar em um controle sustentável para as Arboviroses passa necessariamente pela necessidade de se disponibilizar água em quantidade suficiente, com distribuição e armazenamento adequado para a população, além de uma rede de esgotos capaz de captar e tratar o que for consumido. O saneamento sozinho não é capaz de resolver os problemas dessas Arboviroses, tendo em vista a influências das questões climáticas e ambientais, entretanto, esse consiste em um passo inicial e primordial para a resolução dessas epidemias que assolam a população brasileira, em especial a norteriograndense. A educação em saúde se faz necessário, tendo em vista incutir na população a responsabilidade em cuidar da própria saúde e evitar atitudes que favoreçam a proliferação dos vetores e disseminação das arboviroses.

\section{Considerações Finais}

Observou-se com a pesquisa empreendida que dentre as três Arboviroses abordadas, no Rio Grande do Norte, a Dengue é a que apresenta a maior taxa de prevalência nos últimos 10 anos (2007 a 2016), tendo em vista que a entrada das demais arboviroses aqui abordadas é recente, estando presente em todos os municípios do Estado, apresentando a maior distribuição espacial, principalmente na sétima região, e em regiões climáticas secas, como a quarta e quinta região. Quanto a Zika e Chikungunya, apesar do seu aparecimento recente no Brasil, datando de 2014 os primeiros casos, observamos a sua rápida disseminação em território norteriograndense, estando a Zika presente em 77 municípios e a Chikungunya em 116 municípios do Estado distribuídos em todas as regiões de saúde.

Como limitações do estudo, apontamos as subnotificações e os dados incompletos dos casos de Arboviroses, prejudicando o verdadeiro conhecimento a respeito da prevalência dos casos na população, fazendo com que se subestime os impactos a saúde da população e o consequente despreparo do sistema de saúde para lidar com novos casos e com as parcelas da população mais sujeitas as sequelas deixados pelas infecções. É, portanto, fundamental esclarecer a população a necessidade e a importância da notificação dos casos, para que essas notificações sirvam de base para o preparo e planejamento para se lidar com novos casos de maneira mais eficiente e eficaz. Outra limitação, é a escassez de artigos científico sobre as Arboviroses no Estado.

É de fundamental importância a realização de estudos que busquem conhecer e investigar as Arboviroses no Estado e no Brasil de forma temporal e espacial. A análise retrospectiva das três Arboviroses abordadas, em circulação no território brasileiro e a expectativa das implicações futuras da co-circulação das mesmas, chamam atenção para a necessidade urgente de priorização de medidas governamentais a respeito do controle dos vetores, sendo fundamental para uma melhor avaliação e replanejamento da vigilância epidemiológica do Estado, além de abordagens que envolvam a educação em saúde da população não apenas nos momentos de epidemias, mas constantemente, com vista a reduzir o número de casos anuais. Para tanto, se faz necessário o desenvolvimento de futuros estudos acerca de análises espaciais que possibilitem comparar a distribuição de casos de arboviroses com indicadores como escolaridade, saneamento básico, dentre outros. 
Research, Society and Development, v. 10, n. 7, e10910716220, 2021

(CC BY 4.0) | ISSN 2525-3409 | DOI: http://dx.doi.org/10.33448/rsd-v10i7.16220

\section{Referências}

Alencar, C. H. M., Albuquerque, L. M., Aquino, T. M. F., Soares, C. B., Ramos Júnior, N. A., \& Lima, J. W. O. (2008). Potencialidades do aedes albopictus como vetor de arboviroses no Brasil: um desafio para a atenção primára. Rev Aps, 11(4), 459-67.

Almeida, A. S., Medronho, R. A., \& Valencia, L. I. O. (2009). Análise espacial da dengue e o contexto socioeconômico no município do Rio de Janeiro, RJ. Rev Saúde Públ, 43(4), 666-73.

Almeida, L. S., Cota, A. L. S., \& Rodrigues, D. F. (2020). Saneamento, Arboviroses e Determinantes Ambientais: impactos na saúde urbana. Ciênc. Saúde Coletiva, 25(10), 3857-3868.

Barbosa I.R., Araújo L.F., Carlota F.C, Araújo R.S., \& Maciel I.J. (2012). Epidemiologia do dengue no Estado do Rio Grande do Norte, Brasil, 2000 a 2009. Epidemiol. Serv. Saúde, 21 (1), 149-157.

Barbosa, I. R., Tavares, A. M., Torres, U. P. S., Nascimento, C. A., Moura, M. C. B. M., Vieira, B. V., Araújo, J. M. G., \& Gama, R. A. (2017). Identificação de áreas prioritárias para a vigilância e controle de dengue e outras arboviroses transmitidas pelo Aedes aegypti no município de Natal-RN: relato de experiência. Epidemol. Serv. Saude, 26(3), 629-638.

Brito, A.L. (2015). Perfil epidemiológico da dengue no Brasil, nos anos 2009 a 2013. Trabalho de Conclusão do Curso, UNICEUB-Faculdade De Ciências Da Educação E Saúde, Brasília, DF, Brasil.

Cavalcanti, L. P. G., \& Timerman, A. (2016). Sewage disposal and arboviruses in Brazil. Revista Rene, 17(5), 585.

Comissão de epidemiologia da ABRASCO. (2016). Zika vírus: desafios da saúde pública no Brasil. Rev. Bras. Epidemiol, 19(2), 225-228.

Donalisio, M. R., Freitas A. R. R., \& Zuben A. P. B. V. (2017). Arboviroses emergentes no Brasil: desafios para a clínica e implicações para a saúde pública. Rev. Saúde Pública, 51(30), 1-6.

Fantinato F.F.S.T., Araújo E.L.L., Ribeiro I.G., Andrade M.R., Dantas A.L.M., Rios J.M.T., Silva O. M. V., Silva M. S., Nóbrega R. V., Batista D. A., Leite P. L., Saad E., Percio J., Resende E. A., Souza P.B., \& Wada M. Y. (2016). Descrição dos primeiros casos de febre pelo vírus zika investigados em municípios da região Nordeste do Brasil, 2015. Rev. Epidemiol. Serv. Saúde, 25 (4), 683-690.

Fuck, J. A. B. (2010). Avaliação das ações do programa de controle da dengue em municípios selecionados do oeste catarinense. Rev Saúde Públ, 3(2), 6-19. Honório N.A., Câmara D.C.P., Calvet G.A., \& Brasil P. (2015). Chikungunya: uma arbovirose em estabelecimento e expansão no Brasil, Cad Saúde Pública, 31(5), 906-908.

Instituto Brasileiro de Geografia e Estatística. (2016). Estimativa populacional do Rio Grande do Norte. http://www.ibge.gov.br/estadosat/perfil.php?sigla=rn. Júnior, D. P. F., Serpa, L. L. N., Barbosa, G. L., Pereira, M., Holcamn, M. M., Voltolini, J. C., \& Marques, G. R. A. M. (2019). Vetores de arboviroses no estado de São Paulo: 30 anos de Aedes aegypti e Aedes albopictus. Revista de Saúde Pública, 53(30), 1-11.

Lima-Camara, T. N. (2016). Emerging arboviruses and public health challenges in Brazil. Revista de Saúde Pública, 50(36), 1-7.

Lopes, N., Nozawa, C., \& Linhares, R. E. C. (2014). Características gerais e epidemiologia dos arbovírus emergentes no Brasil. Revista Pan-Amazônica de Saúde, 5(3), 55-64.

Maniero, V. C., Santos, M. O., Ribeiro, R. L., Oliveira, P. A. C., Silva, T. B., Moleri, A. B., Martins, I. R., Lamas, C. C., \& Cardoso, S. V. (2016). Dengue, chikungunya e zika vírus no brasil: situação epidemiológica, aspectos clínicos e medidas preventivas. Almanaque Multidisciplinar de Pesquisa, 1(1), 118-145.

Mussi, R. F. F., Mussi, L. M. P. T., Assunção, E. T. C., Nunes, C. P. (2019). Pesquisa Quantitativa e/ou Qualitativa: distanciamentos, aproximações e possibilidades. Rev. Sustinere, 7(2), 414-430.

Nakagawa, C. K. (2013). Promoção da saúde nas ações de controle e combate ao dengue nas escolas de Ceilândia. Trabalho de Conclusão de Curso, Universidade de Brasília, Ceilândia, Distrito Federal, Brasil.

Secretaria de Saúde Pública. (2016). Boletim Epidemiológico: Atualização da Situação Epidemiológica das Arboviroses no Rio Grande do Norte. 2016. Monitoramento dos casos de dengue, febre de chikungunya e febre pelo vírus zika, até a semana epidemiológica ${ }^{\circ}$ 52/2016. http://adcon.rn.gov.br/ACERVO/sesap/DOC/DOC000000000140313.PDF.

Teich, V., Arinelli, R., \& Fahham, L. (2017). Aedes aegypti e sociedade: o impacto econômico das arboviroses no Brasil. J Bras Econ Saúde, 9(3), 267-276. 